Pacific Journal of Mathematics

THE COMPLETE ENUMERATION OF THE 4-POLYTOPES AND 


\title{
THE COMPLETE ENUMERATION OF THE 4-POLYTOPES AND 3-SPHERES WITH EIGHT VERTICES
}

\author{
Amos Altshuler AND LeON Steinberg
}

\begin{abstract}
The enumeration of 4-polytopes and 3-spheres with eight vertices started in [1], is completed here. We show that there are precisely 624 4-polytopes and 22 non-polytopal 3-spheres with eight vertices that are not quasisimplicial nor pyramidal. We find them all and give a detailed description of the 22 non-polytopal spheres.
\end{abstract}

1. Introduction. Our purpose is to carry out a complete enumeration of the combinatorial 3-spheres and 4-polytopes with eight vertices. One part of this task has been carried out in [1], where all the quasisimplicial 3-spheres and 4-polytopes were enumerated. As mentioned in [1], the pyramidal cases are well known. Thus, to complete the enumeration, we must find all non-pyramidal non-quasisimplicial 3-spheres and 4-polytopes with eight vertices. This is done in the present work. The main result obtained here is:

THEOREM 1. There are precisely 624 4-polytopes and 22 non-polytopal 3-spheres with eight vertices that are not quasisimplicial nor pyramidal.

This theorem, combined with the results obtained in [1] and with former results mentioned in $[1, \S 1]$, yields:

THEOREM 2. There are precisely 1294 4-polytopes and 42 non-polytopal 3-spheres with eight vertices.

As a by-product of the methods used for the enumeration, we get (see $[6, \S 5.5])$ :

THEOREM 3. Every 4-polytope with up to eight vertices is rational. (That is, it is combinatorially equivalent to a polytope in $R^{4}$ all of whose coordinates are rational.)

The present paper should be considered a continuation of [1]. Thus the notation and terminology is that of [1]. In particular, one should 
consult [1, §1] for general background, [1, §2] for the definition of a combinatorial sphere, and $[1, \S \S 4,5]$ for the methods used to prove the non-polytopality of a sphere. All the spheres and 3-complexes in this work are combinatorial, in the sense of $[1, \S 2]$.

The first problem we face-which is perhaps the main problem- is the enumeration of the spheres under consideration. The methods used are completely different from those used in [1], and therefore they are described in detail. The procedures used to determine if a sphere is a polytope are very similar to those implemented in [1] and are described briefly with emphasis only on the differences. In particular, Theorems 3-5 of [1], which served there as the main tool for testing a given 3-sphere for polytopality, are used here too, but in a different way. Here they are used - together with an additional result, namely Theorem 6 in \$4-for an inductive construction of 4-polytopes with eight vertices. Unexpectedly, this process yields all 1294 4-polytopes with 8 vertices. Thus Theorem 6, if known when [1] was written, would have made superfluous the special treatment given to three of the polytopal spheres in $[1, \S 7]$. For another (slight) improvement of [1], see Remark 6 in $\$ 6$.

As in [1], a detailed description of all 646 3-spheres found here is beyond the scope of a single paper. Therefore, we give (in Table 2) a detailed description of only the 22 non-polytopal spheres, and (in Table 3) a brief description of all the 1260 nonpyramidal 4-polytopes with eight vertices. The complete catalogue of 4-polytopes and 3-spheres with eight vertices can be obtained upon request from the second author.

For a brief account of the history of the enumeration of polytopes and spheres one should consult [1, §1]. As mentioned there, Schulz [7] describes 27 non-polytopal 3-spheres with eight vertices, 15 of which are non-quasisimplicial. These 15 spheres are, of course, among the non-polytopal spheres listed in Table 2, and their ordinal numbers in Schulz's paper are given in Table 2 in parentheses.

Our initial goal is the construction of the family $\mathscr{N S}_{8}^{3}$ of all nonpyramidal non-quasisimplicial 3-spheres with eight vertices. Actually, we first construct a family $\mathscr{R}$ of 3-complexes with eight vertices which contains $\mathscr{N} \mathscr{S}_{8}^{3}$ such that $\mathscr{N}_{8}^{3}$ is precisely the family of all spheres in $\mathscr{R}$. The construction of $\mathscr{R}$, in turn, is based upon the construction of certain subcomplexes, called cores, of the members of $\mathscr{R}$. The core of $R \in \mathscr{R}$ is the subcomplex of $R$ composed of the non-simplicial facets of $R$ (and their faces). The construction of the cores is described in $\S 2$, and that of $\mathscr{R}$ in $\S 3 . \mathscr{R}$ turns out to be of cardinality 731 . In $\S 4$ we present Theorem 6, which we use together with Theorems $3-5$ of [1] to construct many 
4-polytopes with 8 vertices from those with 7 vertices. Altogether we obtain 1294 polytopes, among which are all 670 pyramidal and quasisimplicial 4-polytopes with 8 vertices. The boundary complexes of the remaining 624 polytopes are in $\mathscr{N S}_{8}^{3} \subset \mathscr{R}$. Thus there remain 107 members of $\mathscr{R}$ which have to be tested for sphericity and polytopality. This is done in $\S 5$, where we show that among these 107 complexes there are just 22 spheres and none of them is polytopal, thus completing the proof of Theorem 1 . We conclude in $\S 6$ with some remarks.

2. Cores and 8-cores. Let $\mathscr{N}_{8}^{3}$ denote the family of all (combinatorial types of) 3-spheres with 8 vertices which are not quasisimplicial nor pyramidal. In this section we start the construction of the family $\mathscr{N S}_{8}^{3}$. First we give an outline of this construction.

Since each facet in a sphere $S \in \mathscr{N} \mathscr{S}_{8}^{3}$ has at most 6 vertices, it is either a 3-simplex or one of the nine types $A, B, \ldots, I$ depicted in Figure 1.

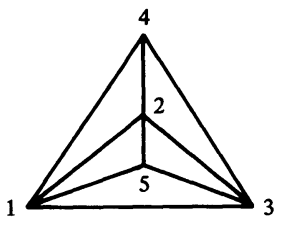

A-12345

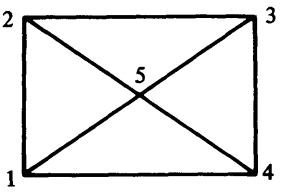

D-12345

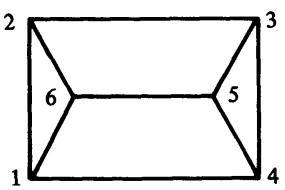

G-123456

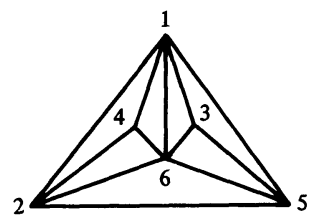

B-123456

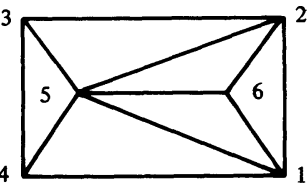

E-123456

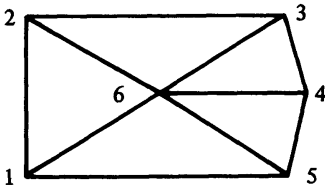

H-123456

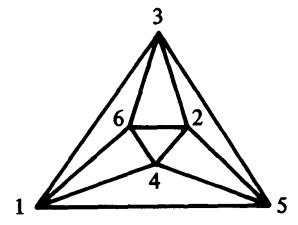

C-123456

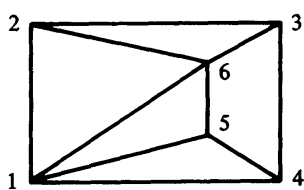

F-123456

Figure 1

The nine 3-polytopes (other than the simplex) with up to six vertices, and their canonic representation. 
Figure 1 also gives a standard representation for each of the nine 3-polytopes by means of the ordered set of its vertices. Note that of these nine polytopes, only $A, B$ and $C$ are simplicial.

Thus each $S \in \mathcal{N S}_{8}^{3}$ is composed, in a natural sense, of "building blocks" which are copies of these ten 3-polytopes. We must combine these building blocks in all possible ways to yield all the spheres in $\mathscr{N S}_{8}^{3}$. Unfortunately, this direct procedure is combinatorially prohibitive. We therefore start by constructing some larger units which we call kernels. Each kernel is composed only of copies of the non-simplicial 3-polytopes with up to six vertices, that is, polytopes of types $D, E, \ldots, I$ (depicted in Figure 1). The kernels are then used as building blocks for still larger units called cores. Each core is then completed, by means of 3-simplices only, to yield a sphere-like complex. To each of these complexes we then apply the operations $A, B$ and $C$ of $[1, \S 3]$ in all possible ways and we obtain a family $\mathscr{R}$ of sphere-like complexes which is large enough to contain all of $\mathscr{N} \mathscr{S}_{8}^{3} \cdot \mathscr{N}_{8}^{3}$ is exactly the family of all the members of $\mathscr{R}$ which are spheres.

Definition 1. An $n$-core is a 3-cell complex $\mathscr{C}$ (see $[1, \S 2])$ with at most $n$ vertices, each of whose 3-cells is a non-simplicial 3-polytope with at most $n-2$ vertices, such that bd $\mathscr{C}$, the boundary complex of $\mathscr{C}$, is simplicial (possibly empty). An $n$-kernel is an $n$-core which is minimal with respect to inclusion.

REMARK. Every $n$-core is clearly the union of pairwise disjoint $n$ kernels, where "disjoint" means they have no common 3-cell. Thus an $n$-kernel is a "prime" $n$-core.

Definition 2. Let $R$ be a 3-cell complex whose boundary complex, bd $R$, is empty. The core of $R$, core $R$, is the subcomplex of $R$ composed of all the non-simplicial 3-cells (facets) in $R$ and their faces. A kernel of $R$ is a non-empty subcomplex of $R$ composed of non-simplicial facets of $R$ (and their faces) whose boundary complex is simplicial, and which is minimal with repsect to inclusion.

Obviously, core $R$ is empty iff $R$ is quasisimplicial; the boundary complex of core $R$ is a simplicial complex (possibly empty); each kernel of $R$ is contained in core $R$; no two kernels of $R$ share a common 3-cell, and core $R$, if not empty, is precisely the union of the kernels of $R$.

If $S$ is a 3-sphere with $n$ vertices which is neither pyramidal nor quasisimplicial, then core $S$ is an $n$-core and every kernel of $S$ is an $n$-kernel. A 
priori it is not clear whether or not every $n$-core $(n$-kernel) is the core (a kernel) of some 3 -sphere. We say that an $n$-core ( $n$-kernel) is genuine if it is combinatorially equivalent to the core (a kernel) of some 3-sphere with $n$ vertices, otherwise it is spurious. One should notice that an $n$-core ( $n$-kernel) is at the same time also an ( $m$-kernel) for every $m \geq n$, but it may be genuine as an $n$-core ( $n$-kernel) and spurious as an $m$-core ( $m$-kernel). However, a genuine $n$-core ( $n$-kernel) with a non-empty boundary is genuine also as an $m$-core ( $m$-kernel) for every $m \geq n$. For $n$-cores it follows from the fact that if $S$ is a 3-sphere with $n$ vertices whose core has a non-empty boundary, then $S$ has at least one simplicial facet, and by a repeated subdivision of that facet one obtains, for every $m \geq n$, a 3-sphere $S^{\prime}$ with $m$ vertices such that core $S^{\prime}=$ core $S$. The reasoning for $n$-kernels is similar. Since in the present work our interest lies merely in 8-cores, and, as we shall see later, each 8-core has a non-empty boundary, no confusion is likely to arise.

For every $S \in \mathscr{N} \mathscr{S}_{8}^{3}$, core $S$ is an 8-core. Thus we would like to construct all the 8 -cores. Since every 8 -core is a union of 8 -kernels, we would like to first construct the family $\mathscr{K}$ of all the 8-kernels. Each 8-kernel is composed of 3-cells of the types $D, E, F, G, H, I$ in Figure 1. Note that the 3-polytope $E$ can be considered as the union, in a natural sense, of a 3-simplex and a 3-polytope of type $D$. Thus if we know the subset $\mathscr{K}^{\prime}$ of $\mathscr{K}$ consisting of all membbers of $\mathscr{K}$ not containing a 3-cell of type $E$, then the rest of $\mathscr{K}$ can be obtained by attaching 3-simplices in all possible ways to all members of $\mathscr{K}^{\prime}$ containing a 3-cell $D$ and whose boundary contains a 2 -simplex of bd $D$-at most one 3-simplex to each such $D$.

Thus, in the construction of $\mathscr{K}^{\prime}$ we use 3-polytopes of types $D, F, G$, $H, I$ only. Each member of $\mathscr{K}^{\prime}$ is obtained by starting with one of these five 3-cells, attaching to a non-simplicial 2-face in its boundary another 3-cell of one of these five types, and repeating the process on the newly generated complexes until we get a 3-complex with a simplicial boundary having 8 or less vertices. We carried out this procedure both by hand and with a computer and obtained 23 8-kernels. They are listed in Table 1 as nos. $1-23$. From this family $\mathscr{K}^{\prime}$ we constructed the members of $\mathscr{K} \backslash \mathscr{K}^{\prime}$ as mentioned above. They are listed in Table 1 as nos. 24-34. Thus $\mathscr{K}$ consists of 348 -kernels which are the "prime" 8-cores. The vertex-set of each of these 8-cores, and of the "composite" 8-cores to be constructed in the next stage, is a subset of $\{1,2, \ldots, 8\}$. For each $i$ we denote by $c_{i}$ the 8-core no. $i$ listed in Table 1. Thus the 8-kernels are $c_{1}-c_{34}$.

For the construction of the "composite" 8-cores, the following lemma is very useful. 
TABLE 1

The 62 8-cores and the number of polytopes, non-polytopal spheres and other members of $\mathscr{R}$ generated from them

\begin{tabular}{|c|c|c|c|c|c|c|c|c|c|}
\hline No. & Facets & $*$ & $* *$ & ${ }_{*}^{* *}$ & No. & Facets & $*$ & $* *$ & ${ }_{*}^{* *}$ \\
\hline 1 & $D_{1}, D_{2}$ & 317 & 16 & 71 & 32 & $G_{2}, E_{9}, D_{7}, D_{8}$ & 1 & & \\
\hline 2 & $\mathrm{H}_{1}, \mathrm{H}_{2}$ & 11 & & & 33 & $G_{2}, E_{10}, D_{7}, \mathrm{D}_{10}$ & 1 & & \\
\hline 3 & $F_{1}, D_{3}, D_{4}$ & 14 & & 1 & 34 & $\mathrm{I}_{4}, E_{7}$ & 3 & & \\
\hline 4 & $F_{1}, D_{3}, D_{5}$ & 23 & & & 35 & $D_{1}, D_{2}, D_{14}, D_{15}$ & 8 & & \\
\hline 5 & $F_{1}, \mathrm{I}_{1}, D_{4}$ & 3 & & & 36 & $D_{1}, D_{2}, D_{14}, D_{16}$ & 13 & & \\
\hline 6 & $F_{1}, \mathrm{I}_{2}, D_{4}$ & 1 & & & 37 & $D_{1}, D_{2}, D_{17}, D_{18}$ & 1 & & \\
\hline 7 & $F_{1}, F_{2}, G_{1}, D_{6}$ & 1 & & & 38 & $D_{1}, D_{2}, D_{17}, D_{19}$ & 1 & & \\
\hline 8 & $F_{1}, F_{3}, F_{4}$ & & & & 39 & $D_{1}, D_{2}, D_{20}, D_{21}$ & 19 & 6 & 8 \\
\hline 9 & $F_{1}, F_{3}, F_{5}$ & 1 & & & 40 & $D_{1}, D_{2}, D_{12}, D_{43}$ & 10 & & \\
\hline 10 & $G_{2}, D_{7}, D_{8}, D_{9}$ & 6 & & & 41 & $D_{1}, D_{2}, D_{27}, D_{44}$ & 2 & & \\
\hline 11 & $G_{2}, D_{7}, D_{9}, D_{10}$ & 1 & & 1 & 42 & $\mathrm{H}_{1}, \mathrm{H}_{2}, D_{22}, D_{23}$ & 1 & & \\
\hline 12 & $G_{2}, I_{3}, D_{8}, D_{9}$ & 1 & & & 43 & $F_{1}, D_{3}, D_{5}, D_{24}, D_{25}$ & 1 & & \\
\hline 13 & $I_{2}, I_{4}$ & 1 & & & 44 & $F_{1}, D_{3}, D_{5}, D_{22}, D_{23}$ & 3 & & \\
\hline 14 & $\mathrm{I}_{4}, \mathrm{I}_{5}$ & 3 & & & 45 & $\mathrm{I}_{4}, \mathrm{I}_{5}, D_{26}, D_{27}$ & 1 & & \\
\hline 15 & $\mathrm{I}_{4}, D_{3}$ & 48 & & 1 & 46 & $\mathrm{I}_{4}, \mathrm{I}_{5}, D_{6}, D_{28}$ & & & \\
\hline 16 & $F_{1}, F_{6}, D_{5}, D_{11}$ & 1 & & & 47 & $I_{4}, D_{3}, D_{25}, D_{29}$ & 3 & & \\
\hline 17 & $F_{1}, F_{7}, D_{4}, D_{11}$ & 1 & & & 48 & $\mathrm{I}_{4}, D_{3}, D_{30}, D_{31}$ & 5 & & \\
\hline 18 & $G_{3}, G_{4}, G_{5}, G_{6}$ & 1 & & & 49 & $I_{4}, D_{3}, D_{26}, D_{27}$ & 1 & & \\
\hline 19 & $G_{7}, G_{8}, D_{8}, D_{9}, D_{12}, D_{13}$ & 1 & & & 50 & $E_{1}, D_{2}, D_{32}, D_{33}$ & 1 & & \\
\hline 20 & $G_{8}, F_{8}, D_{8}, D_{12}, D_{13}$ & 1 & & & 51 & $E_{1}, D_{2}, D_{34}, D_{35}$ & 3 & & \\
\hline 21 & $F_{8}, F_{9}, D_{8}, D_{13}$ & 3 & & & 52 & $E_{1}, D_{2}, D_{17}, D_{18}$ & 1 & & \\
\hline 22 & $F_{8}, F_{10}, D_{8}, D_{13}$ & 1 & & & 53 & $E_{1}, D_{2}, D_{22}, D_{23}$ & 5 & & \\
\hline 23 & $F_{8}, F_{11}, D_{8}, D_{12}$ & 1 & & & 54 & $E_{1}, D_{2}, D_{6}, D_{36}$ & 1 & & \\
\hline 24 & $E_{1}, D_{2}$ & 76 & & 1 & 55 & $E_{1}, E_{3}, D_{17}, D_{18}$ & & & \\
\hline 25 & $E_{1}, E_{2}$ & 1 & & & 56 & $E_{1}, E_{3}, D_{6}, D_{36}$ & 1 & & \\
\hline 26 & $E_{1}, E_{3}$ & 3 & & & 57 & $D_{1}, D_{2}, D_{14}, D_{15}, D_{37}, D_{38}$ & 1 & & \\
\hline 27 & $E_{1}, E_{4}$ & 3 & & & 58 & $D_{1}, D_{2}, D_{14}, D_{16}, D_{39}, D_{40}$ & 1 & & \\
\hline 28 & $F_{1}, E_{5}, D_{4}$ & 1 & & & 59 & $D_{1}, D_{2}, D_{14}, D_{16}, D_{41}, D_{42}$ & & & \\
\hline 29 & $F_{1}, E_{6}, D_{4}$ & 3 & & & 60 & $D_{1}, D_{2}, D_{20}, D_{21}, D_{39}, D_{40}$ & 3 & & 1 \\
\hline 30 & $F_{1}, E_{7}, D_{4}$ & 3 & & & 61 & $D_{1}, D_{2}, D_{20}, D_{21}, D_{41}, D_{42}$ & & & 1 \\
\hline \multirow[t]{2}{*}{31} & $F_{1}, E_{8}, D_{3}$ & 1 & & & 62 & $\mathrm{I}_{4}, D_{3}, D_{30}, D_{31}, D_{45}, D_{46}$ & 1 & & \\
\hline & & & & & & Total & 624 & 22 & 85 \\
\hline
\end{tabular}

The columns $*, * *,{ }_{*}^{*}$ indicate the number of polytopal spheres, non polytopal spheres and other members of $\mathscr{R}$, respectively, generated from the indicated 8-core. The facets are denoted according to the following chart, where the letter indicates the type of facet (according to Figure 1).

$$
\begin{aligned}
& D_{1}-12345, D_{2}-12346, D_{3}-12347, D_{4}-23567, D_{5}-23568, D_{6}-57861, D_{7}-12547, \\
& D_{8}-23658, D_{9}-14637, D_{10}-23657, D_{11}-14785, D_{12}-45876, D_{13}-78213, \\
& D_{14}-15762, D_{15}-15764, D_{16}-15768, D_{17}-58671, D_{18}-58672, D_{19}-58673, \\
& D_{20}-12785, D_{21}-12786, D_{22}-16872, D_{23}-16875, D_{24}-15874, D_{25}-15876, \\
& D_{26}-56781, D_{27}-56783, D_{28}-56873, D_{29}-15872, D_{30}-25871, D_{31}-25873, \\
& D_{32}-15864, D_{33}-15867, D_{34}-35862, D_{35}-35864, D_{36}-56872, D_{37}-23875, \\
& D_{38}-23876, D_{39}-34875, D_{40}-34876, D_{41}-34785, D_{42}-34786, D_{43}-45873, \\
& D_{44}-56784, D_{45}-46871, D_{46}-46873, E_{1}-123457, E_{2}-341268, E_{3}-123468, \\
& E_{4}-234168, E_{5}-341278, E_{6}-143278, E_{7}-123478, E_{8}-235687, E_{9}-364178, \\
& E_{10}-136478, F_{1}-562341, F_{2}-143287, F_{3}-412378, F_{4}-653287, F_{5}-562378, \\
& F_{6}-321478, F_{7}-234187, F_{8}-365214, F_{9}-452178, F_{10}-541287, F_{11}-214587, \\
& G_{1}-265378, G_{2}-136452, G_{3}-235641, G_{4}-135487, G_{5}-235687, G_{6}-126487, \\
& G_{7}-236541, G_{8}-145287, H_{1}-123456, H_{2}-123457, I_{1}-123487, I_{2}-214378, \\
& I_{3}-254178, I_{4}-123465, I_{5}-123478
\end{aligned}
$$


LEMMA 4. Each 8-core contains at most one 8-kernel which is not combinatorially equivalent to $c_{1}$.

Proof. First, we observe that no two 8-kernels, each of which contains a 3-cell with 6 vertices, can be in the same 8-core. If $K_{1}, K_{2}$ are two such 8-kernels in the same 8-core $L$, let $\Phi_{i}$ be a 3-cell with 6 vertices in $K_{i}$, $i=1,2$. Since $L$ has at most 8 vertices, $\Phi_{1}$ and $\Phi_{2}$ must share at least 4 common vertices. These common vertices lie in a 2 -face $\Phi_{3}$ which is a 2-face of both $\Phi_{1}$ and $\Phi_{2}$. Hence $\Phi_{3}$ lies in the intersection of the boundary complexes of $K_{1}$ and $K_{2}$. Since those boundaries are simplicial, $\Phi_{3}$ has just 3 vertices-a contradiction.

Now, of all the 3-cells that occur in our 348 -kernels, $D$ is the only one with less than 6 vertices, and $c_{1}$ is the only 8 -kernel composed of copies of $D$ only.

In view of Lemma 4, the 8-cores composed of two 8-kernels will be obtained by attaching to each of the 348 -kernels a compatible copy of $c_{1}$ in all possible ways. The 8-cores composed of three 8-kernels will be obtained by again attaching to each of these new 8-cores a copy of $c_{1}$ in all possible ways, and so on. One should bear in mind that the resulting complex in each step should have no more than eight vertices.

This procedure was also carried out both by hand and with a computer and yielded 28 "composite" 8-cores, listed in Table 1 as nos. 35-62. Of these, the first 22 are a composites of two 8-kernels, and the remaining six of three 8-kernels. The 8-kernels which comprise each of these 8-cores can be easily read from Table 1 .

In the sequel we will see that $c_{8}, c_{46}, c_{55}, c_{59}$, and $c_{61}$ are spurious while all the others are genuine. That is, there are just 578 -cores, each of which is the core of some $S \in \mathscr{N} \mathscr{S}_{8}^{3}$.

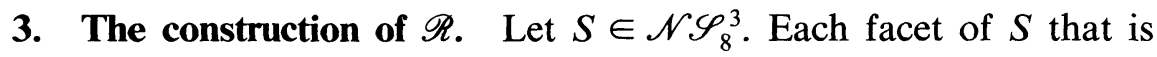
not in core $S$ is either a simplex or of type $A, B, C$ of Figure 1. As in [1, §3], each $A$-, $B$ - and $C$-cell can be simplicially subdivided without adding any new vertices. Carrying out this operation on all the $A$-, $B$ - and $C$-cells of $S$ we obtain from $S$ a 3 -sphere $S^{\prime}$ on the same set of vertices such that core $S^{\prime}=$ core $S$, and all the facets of $S^{\prime}$ that are not in core $S^{\prime}$ are simplices. $S^{\prime}$ is semisimplicial.

Definition 3. A 3-sphere $S$ is semisimplicial if all the facets of $S$ that are not in core $S$ (that is, all the simplicial facets of $S$ ) are simplexes. 
The next stage in the construction of $\mathscr{N S}_{8}^{3}$ is the generation-from the 62 8-cores-of a family $\mathscr{R}^{\prime}$ of 3-complexes on eight vertices which contain all the semisimplicial members of $\mathscr{N S}_{8}^{3}$. Each member of $\mathscr{R}^{\prime}$ is obtained by attaching 3-simplexes to an 8 -core via a procedure resembling that described in more detail in [2].

Let $V=\{1,2, \ldots, 8\}$, and note that none of the 628 -cores has an empty boundary. Let $L$ be some 8-core, and let $a b c(a, b, c \in V)$ be a 2-face in bd $L$. We look for a vertex $d$ in $V \backslash\{a, b, c\}$ such that the union of the 3-simplex $\Delta=a b c d$ (and its faces) and $L$ is a 3-complex $L_{1}$. If bd $L_{1}$ is empty, $L_{1}$ becomes a member of $\mathscr{R}^{\prime}$. Otherwise we choose a 2-face $a_{1} b_{1} c_{1}$ in bd $L_{1}$ and look for a vertex $d_{1}$ in $V \backslash\left\{a_{1}, b_{1}, c_{1}\right\}$ such that the 3-simplex $\Delta_{1}=a_{1} b_{1} c_{1} d_{1}$ is not in $L_{1}$ and the union of $L_{1}$ and $\Delta_{1}$ is a 3-complex $L_{2}$. If bd $L_{2}$ is empty, $L_{2}$ becomes a member of $\mathscr{R}^{\prime}$. Otherwise, we proceed in the same manner. This procedure may fail. A failure occurs if we get a 3-complex $L_{i}$ with non-empty boundary, and there is in bd $L_{i}$ a 2 -face $a_{i} b_{i} c_{i}$ which cannot be completed in the above manner-that is, there is no $d_{i}$ in $V \backslash\left\{a_{i}, b_{i}, c_{i}\right\}$ such that the 3-simplex $\Delta_{i}=a_{i} b_{i} c_{i} d_{i}$ is not in $L_{i}$ and the union of $L_{i}$ and $\Delta_{i}$ (and its faces) is a 3-complex. $\mathscr{R}^{\prime}$ is defined to be the family of all the 3-complexes with eight vertices and empty boundary obtainable in this manner from the 628 -cores. $\mathscr{R}^{\prime}$ obviously contains all the semisimplicial members of $\mathscr{N S}_{8}^{3}$, and it is also clear that every 3-sphere in $\mathscr{R}^{\prime}$ is in $\mathscr{N}_{8}^{3}$.

Using a computer, we constructed the family $\mathscr{R}^{\prime}$. It turned out that $\mathscr{R}^{\prime}$ consists of 240 combinatorially distinct 3-complexes. Thus, the number of semisimplicial spheres in $\mathscr{N S}_{8}^{3}$ is at most 240. The procedure failed completely for $c_{8}, c_{46}, c_{55}$ and $c_{59}$, so these four 8-cores are definitely spurious.

The next stage is the construction of a family $\mathscr{R}$ of 3-complexes with the vertex-set $V$, which contains $\mathscr{R}^{\prime}$ and is large enough to contain all of $\mathscr{N} \mathscr{S}_{8}^{3}$. Each member of $\mathscr{R} \backslash \mathscr{R}^{\prime}$ is obtained from a member of $\mathscr{R}^{\prime}$ by reversing the procedure described in the first paragraph of the present section, that is, by "gluing" together 3-simplices in a member of $\mathscr{R}^{\prime}$ to yield 3-cells of types $A, B$ and $C$. More precisely, $\mathscr{R}$ is defined to be the union of $\mathscr{R}^{\prime}$ and the set of all the 3-complexes obtainable from members of $\mathscr{R}^{\prime}$ by a repeated application of the operations $A, B$ and $C$ of $[1, \S 3]$. It is clear that

$$
\{\text { core } R: R \in \mathscr{R}\}=\left\{\operatorname{core} R: R \in \mathscr{R}^{\prime}\right\} \supset\left\{\operatorname{core} S: S \in \mathscr{N} \mathscr{S}_{8}^{3}\right\},
$$

$\mathscr{N} \mathscr{S}_{8}^{3} \subset \mathscr{R}$ and $\mathscr{N}_{8}^{3}$ is composed of all the members of $\mathscr{R}$ which are spheres. 
The construction of $\mathscr{R}$ is much simplified by the following:

LEMMA 5. No member of $\mathscr{R}$ contains two 6-vertex facets, at least one of which is simplicial.

Proof. Let $R \in \mathscr{R}$ and let $M, N$ be two 6-vertex facets of $R$ such that $N$ is simplicial (that is, of type $B$ or $C$ ). Since $R$ has 8 vertices, $M$ and $N$ must share at least four common vertices, and these must lie in a 2-face of both $M$ and $N$. But this is impossible, since $N$ is simplicial.

Using a computer we constructed the family $\mathscr{R}$ as follows. First, we applied Operation B to all the members of $\mathscr{R}^{\prime}$ in all possible ways. This yielded 13 combinatorially distinct members of $\mathscr{R}$ whose only simplicial facets are a unique type $B$ cell and simplices. Of these 13 complexes, 12 have $c_{1}$ as a core, and one has $c_{39}$ as a core. Denote by $\mathscr{R}_{1}$ the family of these 13 complexes. Next we applied Operation $C$ to all the members of $\mathscr{R}^{\prime}$ in all possible ways and obtained just 3 combinatorially distinct members of $\mathscr{R}$, whose only simplicial facets are a unique type $C$ cell and simplices. Of these three complexes, two have $c_{1}$ as a core and one has $c_{39}$ as a core. Denote by $\mathscr{R}_{2}$ the family of these 3-complexes. Finally we repeatedly applied Operation A to the 256 members of $R^{\prime} \cup \mathscr{R}_{1} \cup \mathscr{R}_{2}$ in all possible ways and obtained the remaining members of $\mathscr{R}$. Altogether, $\mathscr{R}$ consists of 731 combinatorially distinct members.

We now have to determine which of the 731 members of $\mathscr{R}$ are spheres. However, we prefer to delay this task to $\S 5$, since in the next section we will construct, in a completely different way, a certain subset of $\mathscr{N} \mathscr{S}_{8}^{3}$ consisting of polytopal spheres, so that in $\$ 5$ we need only check the remaining members of $\mathscr{R}$ for sphericity.

4. Classification: 624 polytopal spheres. In [1, §6] we quoted from [5] three theorems that played a crucial part in proving that 596 of the 599 polytopal quasisimplicial 3-spheres with eight vertices are indeed polytopal. To these three theorems we now add a fourth, discovered after [1] was sent for publication. This theorem, if used in [1], would have made superfluous the special treatment given in $[1, \S 7]$ to the remaining three polytopal spheres, since their polytopality follows immediately from this new theorem. This theorem is a particular case of a more general theorem stated and proved in [5].

THEOREM 6. Let $Q \subset R^{4}$ be a 4-polytope and let $\Phi_{0}$ be a facet of $Q$ which is a simplex, all of whose vertices are of valence $>4$. Let $\Phi_{1}, \Phi_{2}, \Phi_{3}$, 
$\Phi_{4}$ be the facets $Q$ adjacent to $\Phi_{0}$ (that is, each of them shares a 2-face with $\left.\Phi_{0}\right)$, and let $V=\{1,2,3,4\}$. Then for every $T \subset V$ there is a 4-polytope $P \subset R^{4}$, a projective equivalence $\varphi: Q \rightarrow P$, and a point $x \in R^{4}$ such that $x$ lies beyond the facets $\varphi\left(\Phi_{i}\right)$ of $P, i \in(V \backslash T) \cup\{0\}$, in $\bigcap_{t \in T}$ aff $\varphi\left(\phi_{t}\right)$ and beneath all the other facets of $P$, with respect to $P$, and $\operatorname{vert}(\operatorname{conv}(P \cup\{x\}))$ $=$ vert $P \cup\{x\}$.

This theorem together with the Theorems $3-5$ of $[1, \S 6]$ mentioned above, yield an inductive construction of 4-polytopes (though this is not the way they have been used in [1]): from a 4-polytope with $n$ vertices they yield 4-polytopes with $n+1$ vertices. Thus we programmed these four theorems (the programming was done by I. Shemer, to whom we wish to express our thanks) and applied them to the 31 4-polytopes with 7 vertices. After checking the resulting polytopes for isomorphism, we remained with 1294 combinatorially distinct 4-polytopes with eight vertices. They include the 34 pyramids, the 37 simplicial polytopes and the 599 quasisimplicial 4-polytopes with eight vertices, which were discussed in detail in [1]. There remain 624 4-polytopes with eight vertices. The boundary complexes of these 624 polytopes are of course members of $\mathscr{N S}_{8}^{3}$ and hence also of the set $\mathscr{R}$ of $\S 3$. Thus we need only investigate the 107 remaining members of $\mathscr{R}$ for sphericity and for polytopality.

In the next section we will see that just 22 of those 107 remaining members of $\mathscr{R}$ are spheres, and that none of these spheres is polytopal, thus completing the proof of Theorem 1.

We return to Theorem 6 and to Theorems 3-5 of [1] which yielded the 1294 4-polytopes with eight vertices. From the proofs of those theorems in [5] it can be seen that they are also correct if we replace the Euclidean space $R^{4}$ by the rational space $Q^{4}$. (See [5, Remark 5.2].) Thus, using the notation of these theorems, if $Q$ is a rational polytope, then the polytope $P$ too can be made to be rational. Since the 314 -polytopes with seven vertices are known to be rational (see [6, Exercise 6.5.3]), it follows that the 1294 4-polytopes with eight vertices are rational too, thus proving Theorem 3.

5. The final classification. We still have 107 complexes in $\mathscr{R}$ which have to be tested for sphericity and polytopality. Generally speaking, it is not easy to check whether or not a given 3-complex is a sphere. The unsolved 3-dimensional Poincaré Conjecture stands in the way. However, in our case, where each member of $\mathscr{R}$ has just eight vertices, the problem can be settled rather easily. 
From [2, Theorem 2] it follows that every simplicial 3-manifold with eight vertices is a 3-sphere. It can be easily checked that each of the 8-cores can be simplicially subdivided to yield a simplicial complex without any additional vertices. Thus, from the construction of $\mathscr{R}$ it is clear that each member of $\mathscr{R}$ can be simplicially subdivided, without any additional vertices, to yield a simplicial complex. Hence, it follows that every member of $\mathscr{R}$, which is a 3-manifold, is a 3-sphere. Therefore, one way to check a member of $\mathscr{R}$ for sphericity is to check if it is a 3-manifold. Each of our 8-cores is a connected complex. Thus it follows from the construction of $\mathscr{R}$ that each member of $\mathscr{R}$ is a connected complex. Also, each member of $\mathscr{R}$ has an empty boundary. Thus, to check each member $R$ of $\mathscr{R}$ for being a 3-manifold, all we have to do is to check that the link of every vertex in $\mathscr{R}$ is a 2 -sphere. This is easy to do.

Another way to check the sphericity of a member of $\mathscr{R}$ is to triangulate it without adding any new vertices, and then see if the resulting complex is combinatorially equivalent to one of the 39 simplicial 3-spheres with eight vertices (see [2]). This, too, is easy to do.

It turned out that of the 107 complexes under consideration precisely 22 are spheres. They are listed in Table 2, where the cores of these 22 spheres are also stated. (The cores of the 85 non-spheres can be read from Table 1.) Thus, $\mathscr{N}_{8}{ }^{3}$ has 646 members, as asserted in Theorem 1.

As reflected in Table 1, the only member of $\mathscr{R}$ resulting from $c_{61}$ is not a 3-sphere. Thus $c_{61}$ is spurious. (See also Remark 4 in §6.) Hence, with the four spurious 8-cores found in $\$ 3$ there are altogether five spurious 8-cores, namely $c_{8}, c_{46}, c_{55}, c_{59}$, and $c_{61}$.

Each of our 22 spheres now has to be checked for polytopality. This is done by means of the method developed and described in detail in [1, §5]. In particular, we use Theorem 2 of [1], which asserts that if a 3-sphere has a vertex at which it is not contractible, then it is not polytopal. There are, however, certain differences in the implementation of this method in [1] and here. The differences stem from the fact that the 3-spheres dealt with in [1] had only simplicial facets, while now our spheres also have non-simplicial facets.

Actually, the only non-simplicial facets in the 22 spheres under consideration are 3-cells of type $D$ (that is, quadrangular pyramids). Thus, if $S$ is one of our 22 spheres and $x$ is a vertex in $S$ which is the apex of a type- $D$ cell, then $\operatorname{link}(x, S)$ is not a simplicial complex, as was always the case in [1], since beside triangles it has also quadrangular 2-faces. Thus we can no longer assume, as we did in [1], that if $S$ is polytopal, then it must have a simplicial refill at $x$. 


\section{TABLE 2}

The non-polytopal non-quasisimplicial 3-spheres with 8 vertices

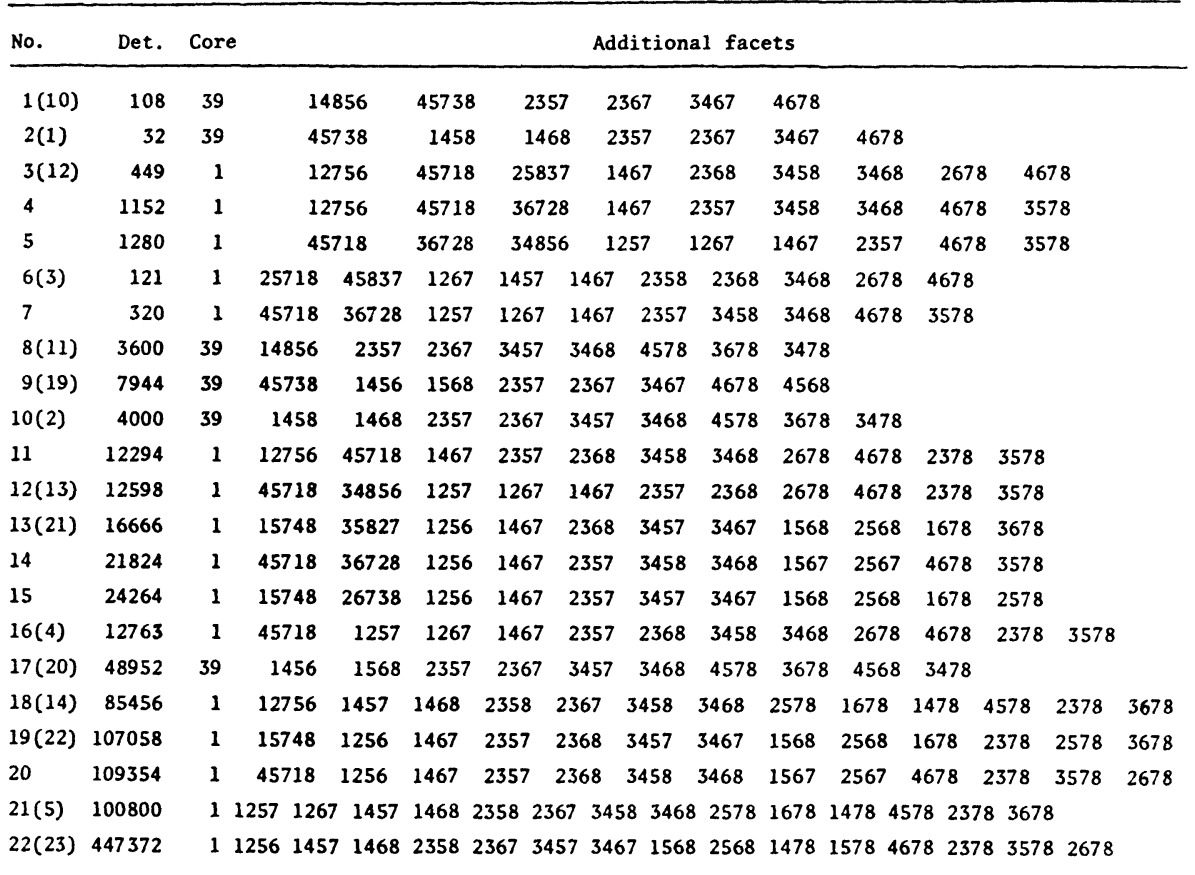

The number in parentheses in the first column is the number of the sphere in Schulz's paper [7]. Det. is the determinant of the edge-valence matrix. In "Additional facets" every 5 -vertex facet is of type $\boldsymbol{A}$. "Core" is the no. of the core of the sphere, as stated in Table 1.

There is, however, an important feature common to our 22 spheres and the 5203 -spheres dealt with in [1, §5]. That is, all the simplicial facets of our 22 spheres are just simplexes and double tetrahedra (type $A$ cells).

Note that the cores of our 22 spheres are just $c_{1}$ and $c_{39}$. Thus the only vertices $x$ such that $\operatorname{link}(x, S)$ is not simplicial, where $S$ is any of our 22 spheres, are just 5 and 6 . In the case that core $S=c_{1}, \operatorname{link}(x, S)$ (for $x=5,6$ ) contains a unique quadrangular 2-face, while in the case where core $S=c_{39}, \operatorname{link}(x, S)$ contains just two quadrangular 2-faces, and they share a common edge. Thus it is clear that if $S$ is polytopal, then in the first case (where core $S=c_{1}$ and $x=5,6$ ) there must be a refill for $S$ at $x$ whose 3-cells consist of a unique type $D$ cell ("covering" the quadrangular 2-face in $\operatorname{link}(x, S)$ ) and simplexes, while in the second case there must be a refill for $S$ at $x$ whose 3-cells consist of either a unique type $F$ cell ("covering" the two quadrangles in $\operatorname{link}(x, S)$ ) and simplexes, or a unique type $G$ cell ("covering" the two quadrangles in $\operatorname{link}(x, S)$ ), a unique type $D$ cell ("covering" the remaining quadrangle in the type $G$ cell) and simplices. 
As examples, we study two cases in detail.

First, let $S$ be the third 3-sphere of Table 2. Then the 2-faces in $\operatorname{link}(5, S)$ are the quadrangle 1234 and the triangles 126, 167, 267, 147, 148, 348, 278, 238. The simplexes 1267, 1478 and 2378 must be in the refill for $S$ at 5 (they form the set $\mathscr{C}$, in the notation of the paragraphs following Theorem 2 in [1]). Thus the boundary complex of ast $(5, S) \cup \mathscr{C}^{\prime}$ consists of the quadrangle 1234 and the triangles 127, 237, 178, 148, 348, 378 (and their faces). The apex of the type $D$ cell that is to cover the quadrangle 1234 is therefore either 7 or 8 . But none of them is possible, since the edges 28 and 47 are interior to ast $(5, S) \cup \mathscr{C}^{\prime}$. Thus $S$ has no refill at the vertex 5 . That is, $S$ is not contractible at its vertex 5 , and therefore $S$ is not polytopal.

Secondly, let $S$ be the first 3-sphere of Table 2. The 2-faces in $\operatorname{link}(5, S)$ are the quadrangles 1234,1278 and the triangles $146,168,468$, 237, 347, 478. The set $\mathscr{C}$ here consists of the 3-simplexes 1468, 3478. Thus the boundary complex of ast $(5, S) \cup \mathscr{C}^{\prime}$ is precisely the boundary complex of the type $F$ cell 872143 . This type $F$ cell cannot be in the refill for $S$ at 5 since edge 47 is interior to ast $(5, S) \cup \mathscr{C}^{\prime}$. Thus $S$ is not contractible at its vertex 5 , and hence it is not polytopal.

In a similar manner we studied all 223 -spheres of Table 2 and found that none of them is contractible at vertex 5 nor at vertex 6 . Thus all 22 of these 3-spheres are not polytopal. This completes the proof of Theorem 1.

15 of the 22 non-polytopal spheres have already been discovered by Schulz [7], and in Table 2 we give in parentheses their ordinal numbers in Schulz's paper.

\section{Remarks.}

1. Table 3 gives a brief description of the structures of the 1260 nonpyramidal 4-polytopes with 8 vertices. For example, it gives the number of facets of each type that occur in our polytopes.

2. Eighty five members of $\mathscr{R}$ turned out to be non-spheres. Each of them has exactly two vertices whose link is not a 2 -sphere. In some cases, the links are 2-manifolds other than 2-spheres (a torus or a projective plane), so these complexes are 3-pseudomanifolds in the sense of [3]. In other cases, the link is a union of two disjoint circuits.

3. It is interesting that in each of the 22 non-polytopal spheres found here, as in each of the other non-polytopal spheres with 8 vertices, there are precisely two vertices at which the sphere is not contractible. (Compare [1, Remark 8.3].)

4. As mentioned in $\S 5, c_{61}$ is a spurious 8-core. In fact, $c_{61}$ cannot be a subcomplex of any 4-polytope. The three quadrangular 2-faces, 1234, 
1278,3478 of $c_{61}$ form a Möbius strip which obviously cannot be geometrically realized in $R^{3}$, while every 2-dimensional subcomplex of the boundary complex of a 4-polytope $P$ is geometrically realizable in $R^{3}$ via a Schlegel diagram of $P$.

\section{TABLE 3}

Classification of the 1260 non-pyramidal 4-polytopes with 8 vertices

\begin{tabular}{|c|c|c|}
\hline $\begin{array}{l}\text { \# of } \\
\text { facets }\end{array}$ & \# of cases & classes of polytopes \\
\hline 6 & $0,0,1$ & 18 \\
\hline 7 & $0,0,3$ & $7,19,21 \mathrm{~A}$ \\
\hline 8 & $0,0,19$ & $\begin{array}{l}2 A^{2}, 3 A^{2}, 5 A, 9,10 A^{2}, 15 A^{3}, 16,20,21,24 A^{3}, 29 A, 30 A, 33,39 A^{2}, 44 A, 45 \\
53 A, 56,62\end{array}$ \\
\hline 9 & $0,5,60$ & $\begin{array}{l}A^{5}(2), A^{3} B(2), A^{3} C, 1 A^{3}(3), 1 A^{4}(2), 1 A B(3), 2 A, 2 A^{2}, 3 A(3), 3 A^{2}, 4 A^{2}(4), 5, \\
10 A, 11,12,14 A, 15 A^{2}(3), 17,21,22,23,24 A^{2}(7), 26 A, 27 A, 28,29,30,32, \\
34 A, 35 A^{2}, 39 A, 39 A^{2}, 39 B, 39 C, 40 A, 40 A^{2}, 44,47 A, 48 A(2), 51 A, 53,53 A, \\
54,60 A\end{array}$ \\
\hline 10 & $0,23,106$ & $\begin{array}{l}A^{5}(3), A^{4}(10), A^{2} B(9), A^{2} C, 1 A^{2}(11), 1 A^{3}(12), 1 B(3), 1 A B(4), 1 A C, 2,2 A, \\
3(2), 3 A(2), 4 A(5), 5,6,10,10 A, 14,15 A(4), 15 A^{2}(9), 24 A(10), 24 A^{2}(9), 25,26, \\
27,29,30,31,34,35 A, 36 A^{2}, 39,39 A(2), 40,40 A, 41,42,43,44,47,48(2), 49, \\
51,53,57,58,60\end{array}$ \\
\hline 11 & $0,73,127$ & $\begin{array}{l}A^{4}(23), A^{3}(21), A^{2} B(13), A^{2} C(4), A B(11), A C, 1 A(10), 1 A^{2}(28), 1 A^{3}(11), \\
1 B(5), 1 C, 2,2 A(2), 3(2), 3 A, 4(2), 4 A(6), 10,13,14,15(2), 15 A(9), 24(5) \\
24 A(14), 26,27,34,35,35 A(2), 36 A(4), 39,39 A(5), 40,40 A, 47,48,50,51 \\
52,53,60\end{array}$ \\
\hline 12 & $0,100,125$ & $\begin{array}{l}A^{4}(17), A^{3}(42), A^{2}(20), A B(14), A C(2), B(4), C, 1(4), 1 A(21), 1 A^{2}(42), 1 B(4), \\
1 C, 2(2), 3,4(3), 10,15(5), 15 A(8), 24(6), 24 A(13), 35(2), 36(3), 36 A(2), 37,38, \\
39(3), 40,40 A\end{array}$ \\
\hline 13 & $0,128,90$ & $\begin{array}{l}A^{3}(56), A^{2}(41), A(9), A B(11), A C(2), B(7), C(2), 1(7), 1 A(40), 1 A^{2}(21), 2,3 \\
4(3), 15(4), 24(7), 35,36(2), 39(2), 40\end{array}$ \\
\hline 14 & $3,109,54$ & $(3), A^{3}(30), A^{2}(57), A(16), B(5), C, 1(14), 1 A(30), 15(3), 24(4), 36,40,41$ \\
\hline 15 & $5,85,27$ & $(5), A^{2}(53), A(26), B(4), C(2), 1(13), 1 A(14)$ \\
\hline 16 & $8,49,8$ & $(8), A^{2}(25), A(24), 1(8)$ \\
\hline 17 & $8,19,4$ & (8), $A(19), 1(4)$ \\
\hline 18 & $6,8,0$ & $(6), A(8)$ \\
\hline 19 & $4,0,0$ & (4) \\
\hline 20 & $3,0,0$ & (3) \\
\hline Total: & $37,599,624$ & \\
\hline
\end{tabular}

$x, y, z$ in "\# of cases" indicates $x$ simplicial polytopes, $y$ quasisimplicial non-simplicial non-pyramidal polytopes, $z$ non-quasisimplicial non-pyramidal polytopes. The symbol $x A^{m} B^{n} C^{l}(r)$ indicates $r$ polytopes with core $c_{x}, m$ cells of type $A, n$ of type $B, l$ of type $C$; a missing $x$ indicates a quasisimplicial polytope, a missing $(r)$ indicates a single polytope. Thus 5 means a single polytope with core $c_{5}$ and simplices, while (5) means five simplicial polytopes. 
5. Although the boundary complex of an $n$-core need not be connected, it is surprising that this occurs for $n=8$. Indeed, the boundary complex of $c_{18}$ is composed of two disjoint 2-spheres, each of which is the boundary complex of a 3-simplex.

6 . In $[1, \S 5]$ we used the easily checked assertion that every 3-polytope with 7 vertices can be simplicially subdivided without the addition of extra vertices. Meanwhile, in [4], it was proved that every polytope of any dimension has a simplicial subdivision with no additional vertices.

7. In the process of constructing the 731 complexes which form the set $\mathscr{R}$, as well as in the construction of the 1294 polytopes described in $\S 4$, we obtained isomorphic copies of the same complex which had to be reduced to a single representative. The determinant of the edge-valence matrix (defined in [1]) proved to be a useful tool for discriminating between non-isomorphic complexes. It proved useful also in the comparison of the polytopes obtained in $\S 4$, and of the members of $\mathscr{R}$. However, among the 1294 4-polytopes with 8 vertices, there are many polytopes which share the same number of facets and the same determinant. Thus we used two more determinants: one is obtained from the edge-valence matrix by replacing all its main diagonal elements by 0 , the other is obtained by replacing them by 1 . For our 1294 polytopes, these three determinants were sufficient for discriminating between any two polytopes having the same number of facets.

8. The 628 -cores were constructed independently by each of the two authors, one by hand and one by computer. Moreover, all the polytopes constructed by means of Theorem 6 and Theorems 3-5 of [1] are in the list of 3-spheres with 8 vertices constructed here and in [1]. It is hoped these checks guarantee that both [1] and the present work are free of major errors.

9. As previously mentioned, 27 of the 42 non-polytopal 3-spheres with 8 vertices are listed by Schulz in [7]. Schulz also proved these 27 spheres to be geometric, in the sense of $[1, \S 2]$. We don't know if the remaining 15 spheres are also geometric.

\section{REFERENCES}

[1] A. Altshuler and L. Steinberg, Enumeration of the quasisimplicial 3-spheres and 4-polytopes with eight vertices, Pacific J. Math., (to appear).

[2] A. Altshuler, Combinatorial 3-manifolds with few vertices, J. Combin. Theory (A), 16 (1974), 165-173.

[3] , 3-Pseudomanifolds with preassigned links, Trans. Amer. Math. Soc., 241 (1978), 213-237. 
[4] The Mani-Walkup spherical counterexamples to the $W_{\nu}$-path conjecture are not polytopal, Math. of Operations Research, (to appear).

[5] A. Altshuler and I. Shemer, Construction theorems for convex polytopes, Israel J. Math., 47 (1.984), 99-110.

[6] B. Grünbaum, Convex Polytopes, Interscience, John Wiley and Sons, 1967.

[7] Ch. Schulz, Nicht-Polytopale 3-sphären mit 8 ecken, Geom. Dedicata, 13 (1982), 325-329.

Received May 9, 1983.

Ben-Gurion University of The NegeV

BEER-SHEVA, ISRAEL

AND

TEMPLE UNIVERSITY

PhiladelPhia, PA 19122 


\section{PACIFIC JOURNAL OF MATHEMATICS EDITORS}

DONALD BABBITT (Managing Editor)
University of California
Los Angeles, CA 90024
CHARLES R. DePrima
California Institute of Technology
Pasadena, CA 91125
R. FinN
Stanford University
Stanford, CA 94305

HERMANN FLASCHKA

University of Arizona

Tucson, AZ 85721

RAMESH A. GANGOLLI

University of Washington

Seattle, WA 98195

ROBION KIRBY

University of California

Berkeley, CA 94720

C. C. MOore

University of California

Berkeley, CA 94720
Hugo RossI

University of Utah

Salt Lake City, UT 84112

H. SAMELSON

Stanford University

Stanford, CA 94305

HaRold STARK

University of California, San Diego

La Jolla, CA 92093

\section{ASSOCIATE EDITORS}
R. ARENS
E. F. BECKENBACH (1906-1982)
B. H. NEUMANN
F. WOLF
K. YoshidA

\section{SUPPORTING INSTITUTIONS}

UNIVERSITY OF ARIZONA

UNIVERSITY OF BRITISH COLUMBIA

CALIFORNIA INSTITUTE OF TECHNOLOGY

UNIVERSITY OF CALIFORNIA

MONTANA STATE UNIVERSITY

UNIVERSITY OF NEVADA, RENO

NEW MEXICO STATE UNIVERSITY

OREGON STATE UNIVERSITY
UNIVERSITY OF OREGON

UNIVERSITY OF SOUTHERN CALIFORNIA

STANFORD UNIVERSITY

UNIVERSITY OF HAWAII

UNIVERSITY OF TOKYO

UNIVERSITY OF UTAH

WASHINGTON STATE UNIVERSITY

UNIVERSITY OF WASHINGTON 


\section{Pacific Journal of Mathematics}

\section{Vol. 117, No. $1 \quad$ January, 1985}

Amos Altshuler and Leon Steinberg, The complete enumeration of the

4-polytopes and 3 -spheres with eight vertices $\ldots \ldots \ldots \ldots \ldots \ldots \ldots \ldots$

Michael James Beeson, The $6 \pi$ theorem about minimal surfaces . . . . . . . . 17

Jeffrey Lawrence Caruso and Stefan Waner, An approximation theorem

for equivariant loop spaces in the compact Lie case ...............27

Jo-Ann Deborah Cohen, Topologies on the quotient field of a Dedekind

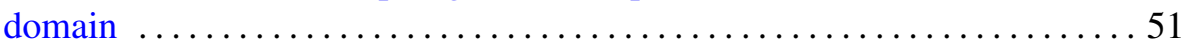

Szymon Dolecki, Gabriele H. Greco and Alois Andreas Lechicki,

Compactoid and compact filters .........................6 69

Roger William Hansell (Sr.), Generalized quotient maps that are

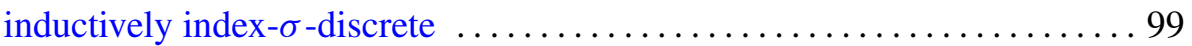

Gerhard Huisken, Capillary surfaces over obstacles $\ldots \ldots \ldots \ldots \ldots \ldots \ldots 121$

Jun Shung Hwang, A problem on continuous and periodic functions . . . . 143

Ronald Fred Levy and Michael David Rice, The extension of equi-uniformly continuous families of mappings $\ldots \ldots \ldots \ldots \ldots \ldots 149$

Kevin Mor McCrimmon, Derivations and Cayley derivations of generalized

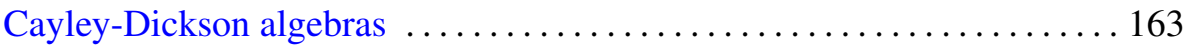

H. M. (Hari Mohan) Srivastava, A multilinear generating function for the Konhauser sets of biorthogonal polynomials suggested by the Laguerre

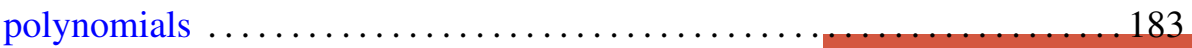

Zhu Jia Lu, Some maximum properties for a family of singular hyperbolic operators 